\title{
韓国鼻科学会会長講演
}

\section{Tissue remodelling in upper airway; epigenetic regulation}

\author{
Heung-Man Lee ${ }^{1,2}$ \\ ${ }^{1}$ The President of Korean Rhinologic Society \\ ${ }^{2}$ Professor, Korea University College of Medicine
}

The pathogenesis of CRS with nasal polyp is unclear, but may involve anatomic abnormalities, local immunologic derangement, and genetic and/or epigenetic factors. Chronic rhinosinusitis with nasal polyps is characterized by both a chronic inflammation and tissue remodelling; as indicated by extracellular matrix protein deposition, basement membrane thickening, goblet cell hyperplasia and subepithelial edema, with reduced vessels and glands. Fibroblasts, which are found in the stroma, are the cellular source of extracelluar matrix (ECM) proteins and are involved in the tissue remodelling of CRS. Tissue remodelling is a critical aspect in all organs which associates matrix production and degradation in reaction to an inflammatory insult leading to a normal reconstruction process or a pathological one. Epigenetics is the study of heritable changes in gene expression that occur without direct changes in the DNA sequence. Epigenetic mechanisms regulate gene expression at the DNA, mRNA, and the chromatin level. Regulation of gene expression occurs at DNA level by DNA methylation and small noncoding RNAs such as microRNAs regulate mRNA translation. Histone modifications result in chromatin remodeling which regulates gene expression. Trichostatin A (TSA) is one of the histone deacetylase (HDAC) inhibitors and induces hyperacetylation of histones. In CRS with nasal polyp, TSA inhibits expression of HDAC2 and increases the hyperacetylation. Inhibition of HDAC2 by TSA reduces TGF- $\beta 1$-induced myofibroblast differentiation and ECM accumulation in nasal polyp-derived fibroblasts. Epigenetic regulations such as inhibition of HDAC2 by TSA can be clinically useful target for inhibition of remodeling in CRS with nasal polyp. 